\title{
El desvelo de la pesadilla Una respuesta a "E1 escepticismo ante las reglas replanteado"
}

Lorena Ramírez Ludeña

Universidad Pompeu Fabra, Barcelona

\section{Introducción}

En su trabajo "El escepticismo ante las reglas replanteado", GUASTINI distingue tres teorías acerca de la interpretación jurídica -el noble sueño, la pesadilla y la vigilia ${ }^{2}$-, que pueden ser caracterizadas por la posición que adoptan con respecto al valor de verdad de los enunciados interpretativos. GUASTINI considera que ninguna de ellas describe adecuadamente el fenómeno de la interpretación en el derecho, dado que no toman en consideración que, en atención a los diferentes instrumentos interpretativos en uso, es posible interpretar de diversos

${ }^{1}$ Publicado en este volumen con traducción de Federico José ARENA. Las citas al texto de GUASTINI que aparecen en este trabajo se corresponden con la versión traducida al castellano. Agradezco a Josep Lluís Martí, Nicola Muffato, Diego Papayannis y Giovanni Ratti sus comentarios a este trabajo.

${ }^{2}$ La distinción entre pesadilla y noble sueño fue introducida inicialmente por HART (1983: 123 y ss.) y, entre nosotros, ha sido desarrollada por Jose Juan MORESO (1997: capítulo 5), quien emplea la noción de vigilia para caracterizar la posición de HART. 
modos un mismo texto. Habría por tanto un marco de interpretaciones posibles ${ }^{3}$. Con ese argumento rechaza las teorías cognoscitivistas: por un lado, rechaza el noble sueño, que sostiene que todos los enunciados interpretativos son verdaderos o falsos; por otro, se opone también a la vigilia, que defiende que hay enunciados interpretativos que son verdaderos o falsos pero también que, con respecto a los casos difíciles, los enunciados interpretativos carecen de valor de verdad. No obstante, su rechazo del cognoscitivismo no le lleva a asumir, como defendería la pesadilla, que cualquier interpretación tiene cabida, sino que hay un marco de interpretaciones posibles entre las que el juez -y cualquier intérprete en general, aunque con efectos distintos- puede optar discrecionalmente, marco fuera del cual se estaría creando derecho, no interpretándolo (p. 46 y ss.) $)^{4}$.

En este trabajo, me centraré fundamentalmente en su reconstrucción de la teoría de la vigilia, que contrasta con la posición de GUASTINI por considerar que determinados enunciados interpretativos son verdaderos. Argumentaré que los defensores de la teoría de la vigilia no tendrían inconveniente en reconocer algunas de las críticas de GUASTINI, al mismo tiempo que logran preservar como rasgo característico de su teoría que hay enunciados interpretativos verdaderos ${ }^{5}$. En otros casos, sostendré que las críticas no son asumibles puesto que, en contra de lo que GuASTINI piensa, su propia concepción conduce a una imagen distorsionada de la práctica jurídica, al poner un énfasis excesivo en el hecho de que, en ocasiones, los diversos instrumentos interpretativos confieren al intérprete la posibilidad de escoger dentro de un marco de posibilidades.

${ }^{3}$ Noción que alude a la teoría de la interpretación de KELSEN (1960: 351 y ss.).

${ }^{4}$ En otras palabras, las tres teorías se diferencian en virtud de si entienden que la interpretación es una actividad cognoscitiva (como suscribiría el noble sueño y parcialmente la vigilia), o decisoria (como defienden los partidarios de la pesadilla en todo caso y los de la vigilia en los casos difíciles).

${ }^{5}$ En sentido estricto, no pueden ser consideradas críticas, puesto que las apreciaciones de GUASTINI son compatibles con lo que es característico de la teoría de la vigilia. 
El desvelo de la pesadilla. Una respuesta....

\section{La teoría de la vigilia}

En los sistemas jurídicos con los que estamos familiarizados existen múltiples instrumentos interpretativos, de los que se sirven los intérpretes para determinar cuáles son las normas del sistema. Éste es un hecho evidente de la práctica difícilmente controvertible, usualmente señalado por GUASTINI, pero no cuestionado por los partidarios de la teoría de la vigilia. El objeto de discusión principal de las diferentes teorías acerca de la interpretación jurídica que se analizarán en este trabajo es precisamente cómo caracterizar adecuadamente este hecho.

Conforme a lo señalado por GUASTINI, la teoría de la vigilia defiende que hay casos fáciles y casos difíciles. En los casos fáciles el significado es claro y con respecto a ellos los enunciados interpretativos (con la forma "T significa M") son verdaderos o falsos. En los casos difíciles, en cambio, el significado es decidido discrecionalmente, careciendo los enunciados interpretativos de valor de verdad (p. 46 y ss.) ${ }^{6}$.

El problema es, según GUASTINI, que la teoría de la vigilia defiende la existencia de casos fáciles y considera marginales los casos difíciles. Ello le conduce a una descripción inadecuada del fenómeno de la interpretación en el ámbito jurídico, puesto que no prestan suficiente atención a los diferentes instrumentos interpretativos y a las diversas normas que pueden ser identificadas mediante su uso. De acuerdo con la posición de GUASTINI, la existencia de múltiples instrumentos supone que el intérprete tiene discreción para optar entre diferentes normas posibles. Esto no implica, sin embargo, que cualquier interpretación sea admisible, sino solo aquellas que están dentro del marco de posibilidades conforme a los diferentes instrumentos interpretativos.

No obstante, si es un hecho constatable que existen numerosos instrumentos interpretativos que conducen a diferentes normas entre las que puede optarse discrecionalmente, cabe preguntarse por qué los defensores de la teoría de la vigilia consideran marginales los casos difíciles. Para GuAstini, ello se debe a que se han centrado en la

${ }^{6}$ Los diferentes instrumentos interpretativos desempeñarían un papel relevante con respecto a este último grupo de casos, esto es, para resolver los casos difíciles. 
interpretación por parte de los jueces y en los problemas de subsunción, esto es, en la interpretación orientada a hechos. No han prestado atención, en cambio, a la interpretación en abstracto, es decir, a la interpretación dirigida a textos, que consiste en identificar las normas expresadas por un texto normativo, sin hacer referencia a un caso concreto (pp. 28-29). Un ejemplo de problema que plantea la interpretación dirigida a hechos, que consiste en subsumir un caso concreto en el campo de aplicación de una norma previamente identificada "in abstracto", sería si la prohibición de entrada de vehículos al parque se aplica o no a las bicicletas (p. 29). Pero, señala GuAstinI, sólo una vez identificada una norma en abstracto -teniendo en cuenta los problemas de ambigüedad que supone el hecho de que los diversos instrumentos interpretativos nos conducen a diferentes normas-, nos preguntamos si un determinado caso cae o no bajo su ámbito de aplicación, es decir, sólo entonces tienen lugar los problemas de interpretación en concreto. Como ejemplo de problema que plantea la interpretación en abstracto, GUASTINI señala que el artículo 13 de la Constitución francesa establece que el Presidente de la República firma los decretos y resoluciones emanados del Consejo de Ministros y se pregunta si hemos de asumir que el Presidente está facultado para firmar o que tiene el deber de hacerlo (p. 29).

Pero, ipor qué los defensores de la vigilia se han centrado en la interpretación orientada a hechos? Según GUASTINI, porque asumen de manera tácita que el significado de los textos jurídicos es parasitario de los lenguajes naturales. En este sentido, los defensores de la vigilia considerarían que los enunciados interpretativos orientados a textos son verdaderos o falsos precisamente porque su valor de verdad depende del lenguaje natural en el que se expresa el derecho ${ }^{7}$. GUASTINI conecta de ese modo la despreocupación por los problemas relevantes en materia interpretativa, que para él son los problemas de ambigüedad de la

7 Y, precisamente porque consideran no problemáticos los enunciados interpretativos orientados a textos, puede afirmarse que la disputa con las diferentes teorías se había centrado en el valor de verdad de los enunciados subsuntivos, y no de los interpretativos en sentido estricto. En cambio, GUASTINI considera que el debate fundamental gira en torno a si hay o no enunciados interpretativos (orientados a textos) verdaderos. 
interpretación en abstracto, con la asunción naive de que el lenguaje jurídico es parasitario del natural, asunción que a su vez supone que la comunicación jurídica entre autoridades e intérpretes es parasitaria de la conversación ordinaria y en gran medida no problemática ${ }^{8}$. En contraste con lo anterior, GUASTINI observa que los jueces no siempre se atienen al significado literal u ordinario y por ello la teoría de la vigilia, si se la entiende como una teoría genuinamente descriptiva, es una teoría falsa o extremamente incompleta (p. 57 y ss.). De hecho, los problemas no vienen dados exclusivamente por los límites que trae aparejados el lenguaje ordinario, sino que la indeterminación depende, según GUASTINI, también de: a) la multiplicidad de métodos interpretativos, que conducen a diferentes normas; b) la dogmática jurídica ${ }^{9}$; c) el sentimiento de justicia de los intérpretes (p. 40).

En definitiva, el principal problema que, conforme a la posición de GuASTINI, enfrenta la teoría de la vigilia es que asume que el lenguaje jurídico es parasitario del natural, lo que le lleva a defender que la interpretación en abstracto no es problemática y a centrarse en la interpretación orientada a hechos. Defienden por tanto que hay un núcleo de significado claro y casos marginales problemáticos que vienen dados por el hecho de que el lenguaje ordinario tiene límites. Según GuASTINI, en cambio, el lenguaje ordinario no determina el valor de verdad de los enunciados interpretativos, sino que existen múltiples instrumentos interpretativos propios del derecho que dan lugar a un marco de posibilidades interpretativas en abstracto. Analizaré los diferentes elementos de la reconstrucción de GUASTINI en los próximos apartados.

${ }^{8}$ Es decir, a diferencia de los casos de discreción, a los que puede dar lugar la solución de casos particulares que sí pueden plantear problemas, la interpretación de textos sería, conforme a los defensores de la vigilia, un acto de conocimiento, no de decisión.

${ }^{9}$ Según GuASTINI (p. 42), todo intérprete se acerca a los textos provisto de una serie de presupuestos teóricos y conceptuales, que condicionan sus interpretaciones, presupuestos que no son otra cosa que las teorías elaboradas por los juristas en un momento lógicamente antecedente a la interpretación de un texto determinado. 


\section{Críticas y réplicas}

Distinguiré tres de las críticas planteadas por GUASTINI a la teoría de la vigilia que, aunque relacionadas entre sí, pueden ser analizadas independientemente. Presentaré asimismo la posición de GUASTINI en cada uno de los casos y, finalmente, defenderé la posición de los teóricos partidarios de la vigilia.

\section{A) La teoría de la vigilia defiende que el lenguaje jurídico es parasitario del lenguaje natural, pero una teoría de la interpretación jurídica no debe asumir una teoría pre-jurídica del significado}

Según GUASTINI, para poder afirmar que determinados enunciados interpretativos son verdaderos o falsos, la teoría de la vigilia debe comprometerse con una teoría del significado que determine su valor de verdad ${ }^{10}$. En este sentido, si determinados enunciados acerca de lo que establecen las disposiciones jurídicas son verdaderos es precisamente porque describen adecuadamente el contenido de las disposiciones, contenido que, según los defensores de la vigilia, viene dado por el sentido ordinario de los términos.

Sin embargo, GUASTINI arguye que, aunque pueda considerarse que ciertas teorías del significado para los lenguajes naturales -como, por ejemplo, la que reconoce un papel central al sentido literal o a las intenciones de quien profiere la expresión-, describen adecuadamente (una parte de) la práctica de adscripción de significado en la conversación ordinaria, tales teorías no suponen una caracterización adecuada de la interpretación en el ámbito jurídico. Los intérpretes del derecho contarían con una serie de herramientas interpretativas para escoger entre un conjunto de significados posibles de un texto jurídico, sin que el sentido ordinario de los términos determine la interpretación correcta. No obstante, GuASTINI señala que lo anterior no supone que

${ }^{10}$ Lo mismo ocurriría con la teoría del noble sueño, que asume que todos los enunciados interpretativos son verdaderos o falsos. 
pueda atribuirse cualquier significado a los textos jurídicos, sino uno de los significados que se encuentran dentro del rango de significados admisibles según el uso común, los métodos interpretativos en uso y las teorías dogmáticas. En este aspecto, como ya he señalado anteriormente, la posición defendida por GUASTINI se diferenciaría de los defensores de la teoría de la pesadilla ${ }^{11}$.

Por tanto, si bien la teoría de la vigilia asume que el lenguaje jurídico es parasitario del lenguaje natural, GUASTINI entiende que una buena descripción del fenómeno interpretativo requiere tener en cuenta la multiplicidad de métodos interpretativos y de otros instrumentos eminentemente jurídicos. El significado ordinario se halla en el mismo nivel que los significados derivados del resto de instrumentos y supone una posibilidad interpretativa más.

Pese a todo, la posición de GuASTINI con respecto al papel que desempeña el lenguaje ordinario no resulta del todo clara. Por un lado, en su crítica a la vigilia defiende que la teoría de la interpretación jurídica no debe asumir una teoría pre-jurídica del significado. Por otro lado, el principal argumento que GUASTINI esgrime contra la teoría de la pesadilla es que la tesis de la inexistencia de un significado previo equivaldría a sostener que ni siquiera el significado literal o prima facie existe antes de la interpretación (p. 52). Añade, no obstante, que cuando una persona atribuye a un texto normativo su significado literal -descartando otros significados disponibles- lleva a cabo una interpretación decisoria, de modo que el significado literal no posee ningún estatus privilegiado con respecto a los demás significados posibles de un texto normativo (n. 68). ¿Entonces, concede GUASTINI

${ }^{11}$ En un sentido trivial, es incorrecto negar, como hace GUASTINI, que el lenguaje ordinario tenga un papel central y previo a cualquier interpretación. Desde la perspectiva de la ciencia jurídica que trata de describir la práctica interpretativa, es necesario conceder un papel relevante al lenguaje ordinario. Así, para comprender adecuadamente la práctica interpretativa debemos compartir en alguna medida -que es difícil de precisar- el sentido que los participantes, los intérpretes, dan a los términos que emplean, lo que nos llevará a interpretar, por ejemplo, sus reivindicaciones con respecto a la intención del legislador según su sentido ordinario. 
algún tipo de papel al significado ordinario, aunque prima facie, o bien éste resulta operativo, junto con el resto de cánones y en el mismo nivel que ellos, cuando se lleva a cabo la interpretación jurídica?

En cualquier caso, las dos posibilidades podrían ser aceptadas por alguien que sostenga la teoría de la vigilia, puesto que ambas son compatibles con defender que hay enunciados interpretativos verdaderos. Así, un partidario de la vigilia podría sostener que hay un significado pre-jurídico -con carácter determinante o solo relevante prima facie- o que la relevancia de éste depende de la práctica jurídica y se halla en el mismo nivel que el resto de instrumentos interpretativos ${ }^{12}$. El verdadero desacuerdo entre la vigilia y GUASTINI radica en asumir -o no- que hay enunciados interpretativos verdaderos acerca del derecho; $\mathrm{o}$, en otras palabras, que hay casos $\operatorname{claros}^{13}$. Lo relevante, entonces, no es si hay una semántica pre-jurídica que condiciona o determina el valor

${ }^{12}$ De hecho el propio HART, principal exponente de la teoría de la vigilia, niega que el significado ordinario determine el contenido de las disposiciones. Según HART, si bien el derecho tiene como objetivo regular la conducta y se sirve de términos generales propios del lenguaje ordinario, elementos como el propósito del legislador pueden desempeñar un papel fundamental en la identificación de las normas. En este sentido, un caso podría ser difícil si, pese a que el significado ordinario es claro, surgen dudas por considerar el propósito del legislador. Por tanto, no parece que los defensores de la vigilia tengan que comprometerse con que los casos difíciles son casos marginales fruto de los límites del lenguaje ordinario. Los casos difíciles serían aquellos sobre los que cualificados juristas desacuerdan, lo que no limita las controversias a los problemas relacionados con el lenguaje ordinario. Véase HART, 1980: 6. Acerca de los inconvenientes que representa para esta forma de entender los casos difíciles el problema del seguimiento de reglas, véase IGLESIAS, 1999: 82 y ss.

13 GuAstini distingue ambas cuestiones y argumenta que, pese a que los enunciados interpretativos carecen de valor de verdad, sí hay casos claros, concediendo por tanto que los enunciados subsuntivos pueden tener, bajo ciertas condiciones, valores de verdad (2010: 126). Sin embargo, si asumimos que los casos claros son aquellos que no resultan problemáticos desde el punto de vista interpretativo, lo serán precisamente porque hay una caracterización de la norma que no es controvertida. Profundizaré en esta cuestión en los próximos apartados de este trabajo. 
El desvelo de la pesadilla. Una respuesta....

de verdad de los enunciados interpretativos, sino si la existencia de diferentes cánones e instrumentos determinan que los jueces siempre tienen discreción ${ }^{14}$.

B) La teoría de la vigilia asume que hay una interpretación correcta en abstracto $y$, sin embargo, las disposiciones son ambiguas en atención a los diferentes instrumentos interpretativos.

GUASTINI afirma que la teoría de la vigilia, precisamente por entender que el lenguaje ordinario determina el valor de verdad de los enunciados interpretativos, se centra en la interpretación orientada a los hechos y no

${ }^{14}$ En cualquier caso, desde mi punto de vista el desarrollo de una práctica jurídica es inconcebible sin una teoría pre-jurídica del significado. Las condiciones de existencia de un sistema jurídico no pueden agotarse en la práctica de identificar textos sin una actitud crítico-reflexiva respecto de ciertos modos admisibles de otorgarles un contenido. Imaginemos que un individuo considera que el Código Civil español es parte del derecho español pero entiende que su significado debe serle atribuido a partir de un programa informático que asigna significados al azar. Imaginemos que otro individuo considera que su contenido depende de lo que su hijo de diez años dice que expresa. Finalmente, un tercer individuo entiende que aquello que expresa el Código Civil depende del lenguaje ordinario. ¿Diríamos que hay un acuerdo entre los diferentes individuos acerca de que el Código Civil es parte del derecho si no hay un acuerdo básico acerca de lo que expresa el Código? Si cada uno, siguiendo su propio criterio, sostiene una interpretación radicalmente diferente de la de los demás respecto de lo que establece el Código Civil, parece que invocar el Código Civil se torna algo superfluo. Dicho en otros términos, si la actividad interpretativa no es constitutiva de la actividad jurídica, la convergencia en las fuentes podría producir los mismos resultados que su ausencia (un total desacuerdo sobre qué normas son válidas) con lo cual podría ser un hecho completamente irrelevante. En este sentido, contra GuASTINI, el propio surgimiento del sistema jurídico lleva aparejados ciertos instrumentos interpretativos de las fuentes, por lo que estos no se desarrollan con posterioridad a su existencia. Esta idea es sugerida por Diego PAPAYANNIS (2010). Por otra parte, como ha señalado recientemente José Juan MORESO en un seminario de la Universidad Pompeu Fabra, el esquema según el cual la 
concede a la interpretación en abstracto el papel que le corresponde. En contraste con esta posición, GUASTINI sostiene que una descripción adecuada de la práctica interpretativa debe reconocer la existencia de numerosos instrumentos interpretativos que suponen que las disposiciones son ambiguas, esto es, que expresan -o que pueden expresar- diversas normas. Dados los diferentes instrumentos interpretativos, que además pueden variar con el tiempo, los jueces tienen discreción para elegir entre las diferentes opciones que integran el marco interpretativo correspondiente ${ }^{15}$. El lenguaje ordinario no desempeñaría por tanto en la interpretación jurídica el papel que le concede la vigilia ${ }^{16}$.

interpretación tiene lugar después de la identificación de las fuentes es inadecuado puesto que si no tuviese lugar alguna interpretación sobre los textos sería difícil considerarlos como jurídicos. Imaginemos que, frente a la necesidad de una nueva fuente de ingresos, el Estado decide que en el Boletín Oficial aparezcan, junto a las disposiciones jurídicas, anuncios de abogados. ¿Cómo diferenciaríamos qué textos son los textos del derecho si no es tomando en consideración su contenido? Acerca del vínculo entre sintaxis, semántica y pragmática, véase GARCíA CARPINTERO, 1996: 9 y ss.

${ }^{15}$ Resulta discutible el papel que GUASTINI asigna a las construcciones de la dogmática como parte del marco interpretativo. En nuestros sistemas jurídicos es innegable que la dogmática desempeña un importante papel. No obstante, se trata de una cuestión contingente, siendo concebible un sistema en que los intérpretes no muestren deferencia a las elaboraciones dogmáticas. Este tipo de supuestos muestra precisamente que, si las construcciones dogmáticas son relevantes a la hora de elaborar el marco interpretativo, es precisamente porque la práctica jurídica las toma en cuenta. No obstante, en un importante sentido el recurso a la dogmática sí es necesario. La palabra "dogmática" es ambigua, pudiendo entenderse como el instrumental teórico básico con el que los juristas ven la realidad del derecho (su esquema conceptual más elemental) o bien como opiniones y construcciones teóricas acerca de los diferentes temas, frecuentemente inspiradas por la política jurídica. En el primer sentido, podría decirse que las elaboraciones dogmáticas forman parte imprescindible del lenguaje de los juristas. Sin embargo, la relevancia de una determinada construcción dogmática es contingente, dependiendo de lo que tomen en consideración los jueces.

${ }^{16}$ Así, GuASTINI considera que la preocupación teórica por el lenguaje ordinario y sus problemas es excesiva. No obstante, las diferentes opciones interpretativas 
Ahora bien, a partir de la constatación del hecho de que en la interpretación jurídica rigen diversos cánones e instrumentos interpretativos, no cabe concluir que los jueces tienen siempre discreción. Así, un defensor de la teoría de la vigilia podría reconocer que hay múltiples instrumentos jurídicos, que estos dependen de la práctica del conjunto de los intérpretes, y que pueden variar con el tiempo en virtud de los cambios en la práctica de dichos intérpretes y, sin embargo, seguir sosteniendo que, con respecto a cada juez individual y desde la perspectiva sincrónica, hay enunciados interpretativos verdaderos. Enfatizar que siempre hay un marco de interpretaciones posibles, dentro del cual el juez tiene discreción, no supone más que una distorsión de la realidad jurídica, que exagera el carácter controvertido de algunos casos que vienen dados y son resueltos en atención a los diferentes instrumentos interpretativos ${ }^{17}$.

Imaginemos que se introduce una regulación que prohíbe la entrada de animales en el metro de Barcelona. Evidentemente, pueden surgir

que vienen dadas por tomar en consideración los diferentes instrumentos interpretativos nos conducen, en el esquema de GuAstinI, a varias formulaciones posibles de la disposición, formulaciones que a su vez deben ser interpretadas conforme al lenguaje ordinario. Y es precisamente por ello, porque las diferentes posibilidades están expresadas haciendo uso del lenguaje ordinario, por lo que tiene sentido reflexionar con carácter general acerca de él y de los problemas que trae aparejados. En definitiva, que haya diferentes instrumentos no comporta que, en última instancia, el lenguaje ordinario no vaya a ser importante. De esta manera, si interpretamos una disposición atendiendo a la intención del legislador, la reformulación estará expresada en lenguaje ordinario y podrá plantear los típicos problemas de vaguedad y textura abierta.

17 Distinguiendo entre la perspectiva individual y la de la comunidad interpretativa, y las perspectivas sincrónica y diacrónica, pero con el fin de defender una concepción realista -a la que denomina "realismo jurídico moderado"-, véase la interesante posición de FERRER BELTRÁn en MORESO Prieto SANChís - Ferrer Beltrán, 2010: 152 y ss. Evidentemente, como FERRER BELtrÁn señala, determinar la comunidad de referencia supone dificultades, pero basta con que el patrón de corrección no dependa de cada intérprete para que el argumento funcione (MORESO - PRIETO SANCHÍs FERRER BELTRÁN, 2010: 153, n. 5). 
dudas respecto de determinados casos fruto de la consideración, por ejemplo, de cuál es el propósito que subyace a la regla. Así, podría afirmarse que la proposición "se prohíbe transportar gatos dentro del metro de Barcelona" es verdadera, aunque puedan generarse dudas con respecto a casos marginales como el acceso de los gatos disecados ${ }^{18}$. La introducción de la normativa ya supone una diferencia práctica con respecto a numerosos supuestos sin que los jueces tengan discreción con respecto a su solución en virtud de los diferentes instrumentos interpretativos. $\mathrm{O}$, en términos más próximos a las posiciones realistas, es innegable que la introducción de esta disposición nos permite predecir cómo se justificará la resolución de un gran número de casos. Asumir que, puesto que hay determinados casos de duda, el juez cuenta con una serie de normas entre las que escoger supone representar el fenómeno de manera mucho más controvertida de lo que en realidad es.

En los casos de duda el juez tiene diferentes posibilidades argumentativas para optar por una de las soluciones. Por ejemplo, en atención al propósito, puede considerar que los gatos disecados están exceptuados de la prohibición. Pero enfatizar que, puesto que se dan estos problemas, la disposición promulgada expresa una multiplicidad de normas posibles nos hace perder de vista que hay un gran número de supuestos regulados que no suscitan dudas. Y, precisamente no suscitan dudas porque hay una caracterización de la norma expresada por la disposición que no es problemática, sin la cual dejaríamos de reconocer que el derecho es un instrumento capaz de guiar la conducta, en alguna medida al menos ${ }^{19}$.

${ }^{18}$ Los escépticos podrían aceptar que la proposición normativa es verdadera, pero negarían que ello presuponga la verdad de un enunciado interpretativo, como defienden los cognoscitivistas. Entre los escépticos que niegan que sea posible un discurso cognoscitivo sobre normas, rechazando la existencia de proposiciones normativas en sentido estricto, véase MAZZARESE (1991).

${ }^{19}$ Como he señalado de manera reiterada, también hay casos difíciles en los que los instrumentos interpretativos desempeñan un importante papel. Pero entender que el juez puede optar entre lo que significa la disposición según el método que apela al significado ordinario, lo que significa conforme a la intención del legislador, lo que significa atendiendo al contexto, etc., supone exagerar el papel de los casos problemáticos y obviar, como expondré a 
El desvelo de la pesadilla. Una respuesta...:

La existencia de una caracterización no problemática de la norma expresada por la disposición puede ser explicada de diversos modos. Así, puede defenderse que en determinados casos no hay interpretación, sino meramente comprensión del contenido de la disposición ${ }^{20}$. O podría defenderse que hay interpretaciones que no generan controversias, pese a considerar que la identificación de la norma requiere propiamente de la actividad interpretativa. En cualquier caso, se requiera o no alguna forma de teorización al respecto, la existencia de una pluralidad de cánones no determina que no haya una forma de entender la disposición en la que los diferentes cánones coincidan. Así, los diferentes instrumentos interpretativos pueden y acostumbran a regular del mismo modo determinados $\operatorname{casos}^{21}$. En cambio, el argumento de GUASTINI pasa de entender que rigen muchos cánones a

continuación, que todos los instrumentos interpretativos generalmente coinciden en regular determinados casos del mismo modo. De hecho, reconstruir como una de las posibilidades de la interpretación en abstracto la que se desprende del propósito de la norma hace que pierda sentido que la regla exista. Si lo relevante en el nivel abstracto es su propósito, ¿qué sentido tiene que el legislador haya introducido la regla en lugar de hacer referencia al propósito? Si las reglas son instancias de otras reglas que las justifican y reducimos el significado de esas instancias a la intención de quien dicta la regla, el significado de su instancia y de su justificación colapsan (SCHAUER, 1991: 113 y ss.).

${ }^{20}$ Esto es, hay una forma de captar una regla que no es una interpretación, sino que se muestra en el acto de obedecerla, en las explicaciones y justificaciones que ofrecemos invocando la regla. En otras palabras, hay una conexión interna entre las reglas y los actos que se manifiesta en la práctica de adoptar la regla como estándar de corrección. Véase MARMOR, 2005: 9 y ss. En cualquier caso, afirmar que hay un núcleo claro de significado que comprendemos no tiene que comprometernos con que el sentido literal es lo único importante, porque una determinada formulación puede parecernos clara en atención al propósito de la norma. La comprensión, que deriva en última instancia del acuerdo en nuestras formas de vida, se muestra en los actos de seguimiento de la regla (WitTGENSTEIN, 1953, sec. 241).

21 Sugiriendo esta idea, Moreso (1997: 222). Estos casos constituirían paradigmas fuertes (BIX, 1993: 56 y ss), que mostrarían la existencia de un consenso con respecto a cómo aplicar los términos y disposiciones. 
defender que no hay enunciados interpretativos verdaderos y que el juez siempre tiene discrecionalidad para decidir entre las diversas interpretaciones. Retomando el ejemplo de los animales en el metro, si nos planteamos, una vez promulgada la disposición, qué es lo que ésta expresa, apreciaremos sin dificultades que los diferentes instrumentos interpretativos coinciden en regular del mismo modo determinados supuestos. Poner el énfasis en que los diferentes instrumentos pueden resolver de manera diversa algunos casos nos hace perder de vista que hay muchos supuestos no problemáticos precisamente porque los diferentes cánones los resuelven del mismo modo. De hecho, probablemente la descripción más adecuada sea que el lenguaje ordinario desempeña un papel fundamental en nuestra comprensión de lo expresado por las disposiciones y, en muchos supuestos, la solución que viene dada por atender al lenguaje ordinario no puede ser controvertida apelando a otros instrumentos, puesto que todos conducen a la misma solución.

Una objeción más general, y tal vez más profunda contra GUASTINI es que su postura no es conceptual, lo que refuerza la idea de que la existencia de diversos cánones interpretativos en los sistemas con los que estamos familiarizados no debe llevarnos a concluir que siempre hay diversas posibilidades interpretativas entre las que el juez puede optar:

1) Que haya diversos instrumentos interpretativos disponibles depende de la práctica y es por lo tanto una cuestión contingente. Podemos imaginar un sistema en el cual no existe semejante complejidad respecto de los instrumentos interpretativos. Digamos que solo se admite un canon interpretativo, por ejemplo el lenguaje ordinario o la intención del legislador, pero no ambos. Supongamos, además, que en caso de duda el juez debe archivar el expediente sin dictar sentencia. En este sistema el juez no tendría discreción.

2) Incluso si, con carácter general, puede afirmarse que un determinado sistema cuenta con múltiples instrumentos interpretativos, y que estos conducen a enunciados interpretativos diversos, pueden haberse consolidado determinados cánones en 
El desvelo de la pesadilla. Una respuesta...

determinados ámbitos de modo que no se dé lugar al margen de discreción que señala GUASTINI ${ }^{22}$. Esto es, aunque pueda afirmarse que en el sistema jurídico con carácter general se emplean diferentes instrumentos interpretativos, puede que con respecto a determinadas disposiciones o en relación con determinados ámbitos no se admitan diversas interpretaciones, es decir, que se haya consolidado una única interpretación como admisible. Esto, evidentemente, puede variar con el tiempo, pero desde la perspectiva sincrónica cabría reconocer que hay enunciados interpretativos verdaderos ${ }^{23}$.

Finalmente, considerar que hay un marco de posibilidades interpretativas no permite concluir que el no cognoscitivismo es verdadero. Esto es, considerar que hay un marco de posibilidades

${ }^{22}$ Esto plantea la cuestión de si la admisión de diversos criterios que determinan el marco depende de cada disposición o del sistema jurídico en general. Así, cabe preguntarse si habría error en caso que, pese a estar consolidada una interpretación, se adopte otra distinta según otro instrumento interpretativo admitido con carácter general para otros preceptos en el sistema jurídico.

${ }^{23}$ Esto mismo parece admitir GUASTINI al sostener que el escepticismo no implica ni presupone que el significado de cada disposición es siempre controvertido. GUASTINI afirma que en la mayor parte de los casos existe, al menos desde el punto de vista sincrónico, una interpretación consolidada y dominante, que produce una norma determinada. Entonces se puede formular la proposición normativa verdadera que afirma la existencia de la norma en ese ordenamiento (GUASTINI, 2010: 134). ¿Cuál sería entonces la diferencia entre su posición y la de un partidario de la vigilia que entiende que, dependiendo de la práctica y de los instrumentos interpretativos que rigen en ella, hay enunciados interpretativos verdaderos? GUASTINI se está limitando a señalar que hay interpretaciones vigentes, lo que no nos llevaría a considerar como error el uso de otros instrumentos interpretativos. Esto, no obstante, no tendría necesariamente que ser negado por un cognoscitivista quien podría reconstruir el fenómeno de dos modos: como tratándose de la interpretación vigente o como un supuesto de restricción del marco de posibilidades interpretativas. Hacerlo de un modo u otro depende de cómo operen las interpretaciones en la práctica y de las actitudes crítico-reflexivas en relación con las mismas. No diferenciar ambos tipos de supuestos oscurece aspectos fundamentales de la práctica interpretativa. 
interpretativas disponibles no tiene que comprometernos con una tesis no cognoscitivista, puesto que seguiría habiendo un enunciado interpretativo, integrado por una disyunción de opciones interpretativas, que sería verdadero. Bajo esta reconstrucción, el propio GUASTINI sería cognoscitivista ${ }^{24}$.

\section{C) La teoría de la vigilia asume que la interpretación relevante se lleva a cabo con respecto a los casos concretos y olvida la trascendencia de la interpretación "in abstracto"}

GUASTINI critica la teoría de la vigilia por centrarse en la interpretación orientada a hechos. Así, en lugar de constatar que hay multiplicidad de instrumentos interpretativos que conducen a normas diversas, la teoría de la vigilia -del mismo modo que la pesadilla y el noble sueño- se centra en los problemas que plantea la interpretación con respecto a los casos concretos $^{25}$. Sin embargo, considero que no cabe formular una distinción

${ }^{24}$ Un aspecto central a considerar es si la aceptación de una determinada interpretación, pese a no estar incluida en el marco de opciones, supone que nos hallamos ante un caso de interpretación incorrecta, incluso si no es revocada, o si la aceptación ex post supone que esa decisión es correcta. Si GUASTINI suscribiese esta última posibilidad, este aspecto nos permitiría distinguir su posición de la de los cognoscitivistas, y lo acercaría a los defensores de la pesadilla. Así parece asumirlo en GuASTINI, 2010: 100. Desde mi punto de vista, en estos casos la disputa sigue siendo interpretativa porque su objeto no es la eficacia que el derecho positivo concede, en muchos ordenamientos, a las decisiones interpretativas de los órganos de aplicación. Se trata de una disputa genuinamente interpretativa, acerca de qué interpretaciones son correctas. Los defensores de la vigilia podrían sostener que la interpretación está fuera del marco, y que por ello está equivocada -pese a reconocer que la decisión es definitiva y que tiene efectos- y además que la práctica puede cambiar de modo que el criterio interpretativo pase a ser correcto en un momento posterior -lo cual no cambiará el hecho de que en el primer momento, cuando la interpretación estaba fuera del marco, dicha interpretación era incorrecta. En este sentido, véase la discusión entre MORESO y Ferrer Beltrán en Moreso - Prieto Sanchís- Ferrer Beltrán, 2010.

${ }^{25}$ Tradicionalmente, los realistas han prestado prácticamente atención exclusiva a la interpretación de los jueces, lo que les ha llevado a una imagen 
tajante entre la interpretación en abstracto (que GUASTINI vincula con problemas de ambigüedad) y la dirigida a hechos (relacionada con problemas de vaguedad). Las cuestiones que debe enfrentar el juez al analizar y resolver los problemas de vaguedad son similares a las que cabría enfrentar cuando se trata de problemas de ambigüedad fruto de la interpretación en abstracto.

Contra lo que señala GuASTINI, no cabe objetar entonces que tradicionalmente se haya olvidado la interpretación en abstracto, dado que los problemas propiamente interpretativos (relativos, como veremos, a casos genéricos) que se plantean en la interpretación orientada a hechos son estructuralmente idénticos a los de la interpretación en abstracto. Esto es, cuando los jueces se enfrentan a problemas con respecto a determinados grupos de casos, el papel que desempeñan los diferentes instrumentos interpretativos al plantear el problema y resolverlo no se diferencia de lo que ocurre en la interpretación en abstracto. E, incluso si aceptamos la distinción entre interpretación en abstracto y orientada a hechos como tajante, ello tiene consecuencias poco plausibles para el modelo defendido por GUASTINI, al comprometerlo con conceder al sentido ordinario de los términos un papel central para diferenciar ambos grupos de casos. Analizaré estas críticas a GUASTINI en los dos próximos apartados.

\section{La distinción entre vaguedad y ambigüedad}

En su trabajo, GUASTINI señala diversas ambigüedades que afectan al término "interpretación". Me centraré en la primera de ellas: la

distorsionada de los problemas interpretativos, que ignora los casos fáciles que no llegan a los tribunales. A su vez, HART parece adoptar una perspectiva básicamente legislativa, centrándose en las causas que dan origen a la indeterminación y no en cómo superarla. No obstante, GUASTINI critica, pese a ser un autor realista, que la vigilia haya puesto el énfasis en la interpretación dirigida a casos, en lugar de en abstracto, que según él es la que requiere mayor atención. Está por tanto criticando la posición de autores como HART precisamente por no adoptar el nivel de abstracción que la interpretación del derecho requiere, lo que tiene cierto aire de paradoja. La crítica de GUASTINI es, sin embargo, comprensible si se toman en consideración las particularidades del realismo genovés, que no limita su atención a la actividad judicial. 
interpretación dirigida a textos (en abstracto) y la orientada a hechos. Según GUASTINI, el término "interpretación" expresa tanto la atribución de significado a un texto como la inclusión de un caso particular en una clase de casos regulados por una norma (p. 27). De esta forma, pese a que en el ámbito de la actividad judicial pueda resultar difícil distinguir los dos casos de interpretación, ambos procesos son lógicamente independientes: atribuir significado a un texto es algo previo y distinto de-aunque relacionado con- la clasificación de casos. En otras palabras, GUASTINI afirma que una cosa es identificar el significado normativo de un texto y otra la interpretación dirigida a hechos, que consiste en subsumir un caso concreto en el campo de aplicación de una norma previamente identificada "in abstracto" (p. 28). Como ejemplo de problema de interpretación dirigida a hechos, GUASTINI señala las dudas que surgen acerca de si la regla "prohibido vehículos en el parque" se aplica o no a las bicicletas. Por otro lado, uno de los ejemplos que GUASTINI señala como supuesto de interpretación dirigida a textos viene dado por el artículo 13 de la Constitución francesa, que establece que el Presidente de la República firma los decretos y resoluciones emanados del Consejo de Ministros, y con respecto al cual se plantea la duda de si el Presidente tiene el poder de firmar o si debe hacerlo (p. 29).

GUASTINI añade que la interpretación dirigida a textos se parece a la traducción puesto que consiste en la identificación del sentido de un texto y en la reformulación del texto interpretado. En cambio, la interpretación dirigida a hechos es una decisión acerca de la extensión de un concepto en relación con un caso concreto. Y, si bien el objeto de la interpretación orientada a textos consiste en enunciados normativos (completos), la interpretación orientada a hechos recae sobre predicados en sentido lógico, es decir, términos que denotan clases. GUASTINI señala, finalmente, que si mediante la interpretación orientada a textos se identifican normas jurídicas (y clases de casos), mediante la interpretación orientada a hechos se contribuye a identificar los casos concretos regulados por cada norma (p. 31).

$\mathrm{Si}$ atendemos a las diferentes consideraciones anteriormente expuestas, la propia reconstrucción de GUASTINI resulta ambigua. En otras palabras, no es fácil determinar cuál es el aspecto determinante 
para trazar la distinción entre la interpretación dirigida a textos y la orientada a hechos.

Así, una lectura podría sugerir que la diferencia entre la interpretación en abstracto y la dirigida a hechos es que la primera toma como objeto los enunciados completos, mientras que la segunda se concentra en una parte de ellos, en particular, en los términos de clase. No obstante, esto no parece ser determinante para mantener la distinción puesto que también pueden plantearse problemas de ambigüedad con respecto a los términos de clase (¿el gravamen sobre la venta de pinturas se aplica a las materias primas o a las obras de arte?).

En una segunda lectura, podría afirmarse que el énfasis está puesto en la diferencia entre interpretar enunciados y determinar qué casos caen bajo su ámbito de aplicación. No obstante, esta segunda lectura nos conduce a dos posibilidades. Puede apuntar a un contraste entre la interpretación de los enunciados en abstracto y: a) la determinación de los casos genéricos que caen bajo su ámbito de aplicación; o b) la determinación de a qué casos concretos, espacio-temporalmente determinados se aplica. A continuación analizaré ambas posibilidades, aunque defenderé que ninguna de ellas hace que la crítica y la posición de GuAstini resulten plausibles.

Aunque GUASTINI enfatiza el carácter abstracto del primer tipo de interpretación, frente al carácter concreto del segundo, si atendemos a los ejemplos que ofrece el aspecto central no parece estar en dicho contraste. Conforme a la reconstrucción de GUASTINI, los problemas que plantea la interpretación con respecto a la inclusión de determinados casos no afectan estrictamente a casos individuales, sino a casos genéricos. En este sentido, las dudas interpretativas acerca de si las bicicletas son vehículos son dudas acerca del caso genérico relativo a las bicicletas, y no a un determinado supuesto delimitado espaciotemporalmente. Y ello es así a pesar de que, desde el punto de vista epistémico, hayamos detectado el problema que suponen las bicicletas porque se ha planteado un determinado caso concreto.

No obstante, asumiré en primer lugar que GUASTINI contrasta la interpretación en abstracto y la determinación de los casos concretos de aplicación. Consideremos entonces que GUASTINI está diferenciando 
entre la interpretación en abstracto -y los problemas que pueda ocasionar- y la interpretación relacionada con su aplicación a supuestos espacio-temporalmente determinados. ¿Por qué la interpretación orientada a hechos (con respecto a la referencia de un término o enunciado) representaría un problema interpretativo distinto al de la interpretación de textos (al de la determinación del sentido de un término o enunciado)? Es cierto que, siguiendo a GUASTINI, ambas actividades son separables y que la primera, la interpretación en abstracto, es previa. Sin embargo GUASTINI no parece tomarse suficientemente en serio que la primera determina la segunda ${ }^{26}$. Si asumimos que el sentido de una expresión u oración es claro, y además conocemos los diferentes aspectos del caso particular -cuestión que tiene que ver con la prueba, y no con la interpretación- no resta por llevar a cabo ninguna actividad interpretativa adicional. Por ejemplo, si al interpretar el término "vehículo" establecemos que un rasgo específico que incluye a las bicicletas resulta fundamental, no habrá ninguna otra actividad interpretativa adicional a llevar a cabo para considerar la inclusión de una determinada bicicleta en el ámbito de aplicación de una norma ${ }^{27}$. Y ello pese a que, evidentemente, calificar un caso concreto como una instancia de "vehículo" constituye una actividad distinta de interpretar en abstracto el término "vehículo"28.

Es más, si atendemos a los ejemplos que introduce GUASTINI, parece que los diferentes supuestos pueden ser reconstruidos indistintamente

${ }^{26}$ El vínculo entre sentido y referencia que aquí se señala es reconocido expresamente por GuAstini (2010: 89, n. 21), quien, sin embargo, no parece tomar en consideración lo que ello conlleva.

${ }^{27}$ A no ser que asumiera que, puesto que estamos prestando atención a cuáles son las obligaciones del juez, en la solución de un caso concreto, haya que atender a otro tipo de consideraciones que no se agotan en la interpretación de las disposiciones (factores pragmáticos en la administración de justicia). No obstante, nada dice GUASTINI a este respecto. Analizando estas cuestiones, Ross: 1958,181 y ss.

${ }^{28}$ Existan o no múltiples criterios interpretativos, el aspecto a destacar es que cada una de las interpretaciones posibles determina los casos de aplicación, sin que reste llevar a cabo una actividad interpretativa adicional al atender a casos concretos. 
El desvelo de la pesadilla. Una respuesta...

como problemas interpretativos relativos a enunciados o a hechos. Esto es, el caso de la inclusión de las bicicletas bajo el ámbito de aplicación de la disposición que contiene el término "vehículo" puede ser reconstruido como un supuesto en que no sabemos si el enunciado "Prohibido vehículos en el parque" expresa la norma relativa a la prohibición de cualquier medio de transporte o solo, por ejemplo, medios de transporte motorizados. Y la problemática relativa a la disposición constitucional ("E1 Presidente firma los decretos") puede ser reconstruida como un supuesto en el que tenemos dudas acerca de si el caso en que el Presidente no quiere firmarlo constituye o no una infracción del precepto. Además, en ambos casos, en el planteamiento de las diferentes posibilidades y en su resolución, el juez cuenta con diversos instrumentos interpretativos. Por tanto no parece que en la tarea del juez la interpretación orientada a hechos sea una actividad esencialmente distinta de la interpretación en abstracto.

De todos modos, no defenderé que los casos de ambigüedad son reconducibles a casos de vaguedad, ni que ambos grupos de problemas no puedan distinguirse con carácter general. Asumiré simplemente que, por lo que respecta a la actividad interpretativa en el ámbito jurídico, los problemas de vaguedad pueden ser siempre reconstruidos como problemas en que tenemos dudas acerca de qué norma expresa una determinada disposición. GUASTINI afirma en cambio que la interpretación dirigida a textos es previa y más importante que la dirigida a hechos, que solo supondría problemas en los casos difíciles. Precisamente por ello critica la atención preferente puesta en los casos de vaguedad. No obstante, como ya he señalado, en mi opinión los problemas interpretativos que representa la vaguedad de un término de clase son estructuralmente idénticos a los que plantea la ambigüedad de las disposiciones. Un jurista que reflexiona en torno a un problema de vaguedad respecto de un término de clase se hace los mismos planteamientos que si se tratara de un problema de ambigüedad. En este sentido, al hacer referencia a la ambigüedad GUASTINI distingue diferentes situaciones: en ocasiones dudamos si una disposición expresa $\mathrm{N} 1$ o N2, en ocasiones tenemos claro que expresa N1 pero dudamos de 
si también expresa N2, etc. Pues bien, los problemas que se plantean frente a supuestos de vaguedad son fácilmente reconducibles a supuestos en que dudamos si una disposición expresa N1 o N2. Por ejemplo, el caso de la prohibición de la entrada de bicicletas en el parque deja constancia de nuestras dudas acerca de si la disposición expresa una norma en virtud del sentido ordinario de los términos (N1: "Está prohibido que entre al parque todo aquello que sea un vehículo conforme al uso ordinario") o en virtud de la intención del legislador (N2: "Todos los vehículos que provoquen los daños que el legislador quería evitar no pueden entrar"). ¿Por qué no pueden caracterizarse entonces los problemas de vaguedad como problemas en que tenemos dudas acerca de qué norma expresa la disposición?

\section{El papel del lenguaje ordinario}

Incluso si se rechaza que cualquier problema de vaguedad en sede de interpretación judicial puede ser reconstruido como un problema de ambigüedad del texto jurídico, GUASTINI tendría dificultades para distinguir ambos tipos de indeterminación sin recurrir al significado ordinario. Afirma que la teoría de la vigilia ni siquiera distingue entre dos tipos de cuestiones, "(a) si el enunciado normativo 'No se admiten vehículos en el parque' se aplica a las patinetas o los triciclos, y (b) si el mismo enunciado se aplica a ambulancias o coches de bomberos. Estas cuestiones, sin embargo, son de naturaleza bien diferente. La pregunta sobre las patinetas y los triciclos se refiere a la extensión del predicado 'vehículo' y es, por lo tanto, una cuestión de interpretación 'in-concreto' dirigida a hechos (de clasificación de hechos). La dificultad se presenta dado que no es pacífico que las patinetas o los triciclos sean vehículos en cuanto bien podrían, en cambio, ser juguetes. Mientras que la cuestión acerca de las ambulancias y los coches de bomberos es una cuestión de interpretación 'in abstracto' dirigida a textos y referida a la identificación misma de la norma. Ello en cuanto las ambulancias y los coches de bomberos son, desde todo punto de vista, vehículos" (p. 55 y ss.). No obstante, no resulta claro cómo podría trazarse la distinción 
El desvelo de la pesadilla. Una respuesta...

entre ambos grupos de $\operatorname{casos}^{29}$ si no es reconociendo que el significado ordinario desempeña un importante papel para formular la propia distinción: si se trata de casos marginales, que presentan dudas en relación con el sentido ordinario, estaremos frente a un problema de vaguedad; en cambio, será un problema de ambigüedad si constatamos que, junto al sentido ordinario, hay otras posibilidades interpretativas. Si no tomamos en consideración el sentido ordinario, ¿por qué el caso de los triciclos no podría representar un problema de interpretación en abstracto, en virtud del cual tenemos dudas acerca de si la disposición expresa la norma que tiene en cuenta el sentido ordinario o la interpretación del legislador? Conforme a la intención del legislador el caso podría estar claramente excluido del ámbito de aplicación de la regla y no plantear problemas de vaguedad.

\section{Consideraciones finales}

GUASTINI señala que en los sistemas jurídicos existen diversos instrumentos interpretativos, que son además cambiantes. Los diferentes instrumentos suponen que las disposiciones no expresan una única norma sino una multiplicidad de ellas, entre las que el intérprete puede optar. Quien interpreta adscribe por tanto un significado a un texto, entre otros posibles, por lo que los enunciados interpretativos carecen de valor de verdad. Precisamente por ello, según el propio GUASTINI, no puede considerársele como un partidario de la vigilia, aunque tampoco de la pesadilla, dado que postula la existencia de un marco de posibilidades fuera del cual habría creación.

En este trabajo, he cuestionado algunos de los puntos anteriores. $\mathrm{He}$ defendido que, en muchos aspectos, su posición es cercana a la de los partidarios de la vigilia, existiendo simplemente diferencias en cuanto al énfasis con respecto a los casos problemáticos. No obstante, considero que algunos elementos centrales en su reconstrucción lo convierten en un claro partidario de la pesadilla.

${ }^{29}$ Ambos son casos de subsunción genérica conforme a la distinción de AlchourRón y BulYGin (1991: 303 y ss.). 
Hay tres aspectos que parecen alejar a GUASTINI de la vigilia: el carácter adscriptivo de la interpretación, su naturaleza cambiante y la existencia de múltiples instrumentos interpretativos. Sin embargo, si bien considero que la naturaleza adscriptiva de la interpretación y su carácter cambiante no pueden cuestionarse, creo también que no nos permiten concluir que los enunciados interpretativos carecen de valor de verdad. Tampoco nos conduce a tal conclusión el reconocer que existen múltiples instrumentos interpretativos.

Es comúnmente aceptado que las palabras no están vinculadas de manera natural con sus significados ${ }^{30}$. Sería extraño cuestionar que la conexión entre ambos elementos es arbitraria y depende de que exista una práctica de atribución de significado -ya sea por relacionar la palabra con un sentido o por emplearla para hacer referencia a determinados objetos. Sin embargo, lo anterior no nos conduce a negar que determinados enunciados, con la forma "T significa S" sean verdaderos o falsos. Lo serán en virtud del uso general que se haga del término y no de cada individuo en particular ${ }^{31}$.

De modo similar, puede defenderse que las prácticas interpretativas en el ámbito jurídico -que, como señala GUASTINI, presentan ciertas peculiaridades- determinan el valor de verdad de los enunciados interpretativos. Si bien con carácter individual los diferentes intérpretes no determinan las interpretaciones correctas de los textos, la práctica en su conjunto sí las determina. En este sentido, aunque un juez individual podría equivocarse -lo que no obsta a que su interpretación pueda acabar

${ }^{30}$ Incluso aquellos que entienden que descubrimos los significados niegan que las palabras estén ligadas a esos significados con independencia de que haya una práctica en que se usan las palabras para referirse a ciertos objetos. En este sentido, véase KRIPKE, 1980 y PUTNAM, 1975.

${ }^{31}$ Evidentemente este punto podría ser cuestionado por quienes defienden una semántica acorde con las apreciaciones de los llamados "nuevos teóricos de la referencia", que entienden que el significado de los términos puede trascender a la comunidad en su conjunto. Sin embargo, en este trabajo he prescindido de tales consideraciones puesto que intento presentar como intuitiva una posición que, compartiendo los rasgos centrales de las tesis de GUASTINI, no extraiga en cambio sus conclusiones. 
teniendo consecuencias jurídicas- la comunidad interpretativa en su conjunto no puede hacerlo, puesto que ella determina los criterios de corrección interpretativa ${ }^{32}$.

Además, a efectos de caracterizar adecuadamente nuestras intuiciones compartidas acerca del lenguaje en general y de la interpretación jurídica, es importante tomar en consideración dos perspectivas: la sincrónica y la diacrónica. Resultaría extraño discutir que la comunidad interpretativa puede hacer que lo que antes (en el momento t1) era una interpretación correcta, ahora (en el momento t2) no lo sea. En este sentido, desde la perspectiva diacrónica las interpretaciones correctas pueden variar, lo que no impide que hagamos referencia a casos de error e interpretaciones correctas en cada uno de los momentos (esto es, si atendemos a la perspectiva sincrónica). Así, aunque haya cambios en las interpretaciones correctas, esto no varía la forma de analizar las interpretaciones previas, que dependían de las consideraciones de la comunidad en su momento. En relación con lo anterior, si bien desde el punto de vista sincrónico hay enunciados interpretativos verdaderos y los intérpretes individuales pueden equivocarse, desde la perspectiva diacrónica los instrumentos interpretativos pueden variar, si se producen los cambios correspondientes en la comunidad interpretativa.

$\mathrm{Si}$, a partir de la naturaleza adscriptiva y cambiante de la interpretación no se puede concluir que los enunciados interpretativos carecen de valor de verdad, cabe preguntarse si podemos llegar a esa conclusión tomando en consideración el otro de los aspectos destacados por GUASTINI: que, desde la perspectiva sincrónica, existen múltiples instrumentos interpretativos disponibles. En este trabajo he presentado diversos argumentos en contra de considerar que la existencia, en los

32 Esta misma idea es sostenida por Coleman y Leiter (1995: 252), quienes distinguen entre objetivismo fuerte y mínimo, atendiendo a si las interpretaciones correctas pueden trascender a los participantes. En otras palabras, podríamos decir que hay una dependencia constitutiva de las creencias y actitudes de los miembros de una comunidad de intérpretes, lo que no supone que las interpretaciones correctas dependan de lo que considere cada uno de ellos. O, en términos de SEARLE, se trata de un fenómeno ontológicamente subjetivo pero epistémicamente objetivo (1995: 31 y ss.). 
sistemas con los que estamos familiarizados, de múltiples instrumentos interpretativos nos conduzca a que los enunciados interpretativos carecen de valor de verdad. En este sentido he sostenido que: a) la existencia de múltiples instrumentos es en todo caso contingente, puede haber sistemas que carezcan de ellos; b) normalmente existen diferentes instrumentos, pero con respecto a determinados ámbitos o en relación con algunas disposiciones sólo se admite una interpretación; c) incluso en aquellos casos en que podríamos decir que los diferentes instrumentos desempeñan un papel, si atendemos a ellos hay un núcleo de supuestos regulados del mismo modo, que no resultan controvertidos. Pasar de destacar algo importante como es que hay diferentes instrumentos, a enfatizar que hay un marco de opciones entre las que el intérprete puede optar, como hace GUASTINI, supone exagerar el carácter problemático de algunos supuestos. Que en el sistema en su conjunto haya diferentes instrumentos no quiere decir que sea admisible emplearlos con respecto a cada una de las disposiciones y en cada uno de los casos. En otros términos, la existencia de casos difíciles no tiene incidencia en la solución de casos fáciles. En este sentido, su énfasis en la interpretación en abstracto y en la existencia de diferentes posibilidades interpretativas en virtud de los diferentes instrumentos nos conduce a una imagen distorsionada de la práctica. Sin embargo, incluso concediendo que las disposiciones expresan diversas normas en atención a los diferentes instrumentos, no habría obstáculo para considerar que GUASTINI es un autor cognoscitivista, precisamente porque su concepción daría cabida a la existencia de enunciados interpretativos que, recogiendo una disyunción de interpretaciones posibles en virtud de los diferentes instrumentos interpretativos, serían verdaderos ${ }^{33}$.

Como señalé al comienzo del trabajo, es un hecho fácilmente constatable de las prácticas jurídicas con las que estamos familiarizados que hay diversos instrumentos empleados por los intérpretes. La cuestión es, no obstante, qué lectura hacemos de estos hechos. Dicha

${ }^{33}$ No obstante, en el esquema de GuASTINI estos enunciados no tendrían un carácter adscriptivo sino meta-interpretativo (esto es, descriptivo de interpretaciones de otros), por lo que no serían genuinamente interpretativos. 
interpretación estará evidentemente condicionada por la teoría del derecho que se defienda y por cómo se entienda la labor del teórico que trata de reconstruir el fenómeno interpretativo. Por ejemplo, DwORKIN entiende que la práctica jurídica es una práctica argumentativa en que el propósito es la justificación de la coerción estatal. En su reconstrucción, los diferentes instrumentos interpretativos se emplean para encontrar la respuesta correcta. Y, para conocer cómo se emplean los instrumentos interpretativos y ser capaz de identificar la respuesta correcta, es necesario, afirma DWORKIN, ser un participante de la práctica. GUASTINI no fundamenta en este trabajo su interpretación de los hechos prominentes de la práctica como plasmando diversas opciones interpretativas -en lugar de, por ejemplo, defender que hay una respuesta correcta. Defiende que las disposiciones expresan múltiples normas conforme a los diversos instrumentos interpretativos, entre las que pueden optar los intérpretes, y que las diversas alternativas pueden ser descritas por el teórico. Hay por tanto un conjunto de interpretaciones posibles con respecto a las cuales el juez tiene discreción. En este punto, como he intentado destacar a lo largo del trabajo, la posición de GUASTINI se acerca a la de muchos positivistas de corte hartiano, puesto que el juez tiene un margen de discreción que viene dado por la propia práctica y además hay respuestas que no son correctas. Sin embargo, a diferencia de la vigilia, GUASTINI destaca la existencia de una pluralidad de instrumentos, que ofrecen al intérprete un conjunto de posibilidades interpretativas y que impiden hablar de enunciados interpretativos verdaderos. Como he tratado de mostrar, enfatizar que hay múltiples criterios que determinan diversas normas en abstracto nos conduce a una caracterización de la práctica jurídica que distorsiona el hecho de que contamos con numerosos casos no problemáticos, pero esto no convierte a GUASTINI en un no cognoscitivista. GUASTINI podría ser considerado un defensor del cognoscitivismo siempre que reconozca que los enunciados que recogen la disyunción de interpretaciones posibles son verdaderos y defienda además que, fuera del marco, hay interpretaciones incorrectas. En tal caso, GUASTINI sería un defensor, aunque pesimista, de la vigilia. No obstante, rechaza que haya enunciados interpretativos verdaderos enfatizando la noción de vigencia, 
lo que parece conducirle a que atender a opciones fuera del marco o considerar posibilidades distintas de la que se ha consolidado en la práctica no constituyen casos de error en sentido estricto. Pero entonces la posición de GUASTINI no puede distinguirse de la pesadilla y su énfasis en la existencia de una pluralidad de interpretaciones posibles se torna superfluo en el debate con la vigilia ${ }^{34}$. GUASTINI no deja de ser, pese a sus intentos, un defensor sofisticado de la pesadilla.

\section{Bibliografía}

Alchourrón, C. y Bulygin, E., 1991: Análisis lógico y derecho. Madrid: Centro de estudios constitucionales

BIX, B., 1993: Law, language and legal determinacy. Oxford: Oxford University Press

Coleman, J. y Leiter, B., 1995: "Determinacy, objectivity, and authority", en MARMOR, 203-278

GarcíA-CARpintero, M., 1996: Las palabras, las ideas y las cosas. Barcelona: Ariel

GuAstini, R., 2010: Nuevos estudios sobre la interpretación. Bogotá: Universidad Externado de Colombia

GuAstini, R., 2011: "Rule-scepticism restated", en Green, L. - LeIter, B.: Oxford Studies in Philosophy of Law, vol. 1. Oxford: Oxford

${ }^{34}$ Esto es, el aspecto central del debate con la vigilia no parece ser si hay o no convergencias interpretativas y si estas son o no frecuentes. Si GuASTINI considera que no hay error aunque se opte por una interpretación fuera del marco, o por una distinta de la que se había consolidado -puesto que un juez, sobre todo si es de última instancia, puede apartarse de la interpretación dominante- es un claro exponente de la pesadilla. Dejaría constancia de ello el aceptar que una interpretación que en principio podría ser considerada como error no lo es si con posterioridad se genera el consenso adecuado. Pero defender lo anterior parece desvirtuar distinciones que son importantes en el esquema de GUASTINI, por ejemplo el contraste entre interpretación y creación. Sólo cabría entonces hablar de error en el sentido -débil- de que es probable que en el futuro los tribunales se aparten de la decisión. Defendiendo esto último, Ross, 1958: 77. 
El desvelo de la pesadilla. Una respuesta...

University Press. Citado por la traducción de ArENA, F., 2011: "E1 escepticismo ante las reglas replanteado". En este número.

HART, H., 1983: Essays in Jurisprudence and philosophy. Oxford: Oxford University Press

HaRT, H., 1980: "El nuevo desafío del positivismo jurídico". Sistema, 36: $3-18$

IGLESIAS, M., 1999: El problema de la discreción judicial. Madrid: Centro de Estudios políticos y constitucionales

KeLSEN, H., 1960: Teoría pura del derecho, $2^{\mathrm{a}}$ ed. Citado por la traducción de Vernengo, R., 1982. México: UNAM

KRIPKE, 1980: Naming and necessity. Cambridge, Mass: Harvard University Press

MARMOR, A., 2005: Interpretation and legal theory, $2^{\mathrm{a}}$ ed. Oregon: Hart Publishing

MARMOR, A., 1995: Law and interpretation. Oxford: Oxford University Press

MAZZARESE, T., 1991: “Norm proposition': epistemic and semantic queries". Rechtstheorie, 22: 39-70.

MoRESO, J., 1997: La indeterminación del Derecho y la interpretación de la Constitución. Madrid: Centro de estudios políticos y constitucionales

Moreso, J. - Prieto Sanchís, L. - Ferrer Beltrán, J., 2010: Los desacuerdos en el derecho. Madrid: Fundación coloquio jurídico europeo

PAPAYANNIS, D., 2010: "El aspecto interpretativo de la regla de reconocimiento". Manuscrito inédito

Putnam, H., 1975: "The meaning of "meaning"”, en Mind, Language and reality, Philosophical papers. Vol. 2. Cambridge: Cambridge University Press, 215-71

Ross, A., 1958: On Law and Justice. Londres: Stevens \& Sons. Citado por la traducción de CARrió, G., 2005: Sobre el Derecho y la Justicia. Buenos Aires: Eudeba 
SCHAUER, F., 1991: Playing by the rules. Oxford: Oxford University Press. Citado por la traducción de ORUnesu, C. - Rodriguez, J., 2004: Las reglas en juego. Barcelona: Marcial Pons

SEARLE, J., 1995: The construction of social reality. Nueva York: Simon and Schuster

Wittgenstein, L., 1953: Investigaciones filosóficas. Citado por la traducción de GarcíA, A. - Moulines, U., 2008. Barcelona: Crítica. 